\title{
Indoor Surveillance Security Robot with a Self-Propelled Patrolling Vehicle
}

\author{
Hou-Tsan Lee, Wei-Chuan Lin, and Ching-Hsiang Huang \\ Department of Information Technology, Takming University of Science and Technology, No. 56, Sec.1, Huanshan Road, Neihu, \\ Taipei 11451, Taiwan \\ Correspondence should be addressed to Hou-Tsan Lee, houtsan@takming.edu.tw
}

Received 10 March 2011; Revised 28 July 2011; Accepted 29 July 2011

Academic Editor: Ali Meghdari

Copyright ( $\odot 2011$ Hou-Tsan Lee et al. This is an open access article distributed under the Creative Commons Attribution License, which permits unrestricted use, distribution, and reproduction in any medium, provided the original work is properly cited.

Self-propelled patrolling vehicles can patrol periodically in the designed area to ensure the safety like men do. The proposed vehicle cannot only save manpower, but also ensure the performance without mistakes caused by man. It is different from the traditional patrolling system which is limited by the manpower and the fixed camera positions. To improve such situation, this paper proposes a self-propelled patrolling vehicle which can move automatically to a wider range and record the monitored image by IPCAM within a predefined patrolling route. Besides, the user can use the mobile device or website to connect to the vehicle at anytime and anywhere and control it to move to the position to get the indoor image user wants. The position of self-propelled vehicles can be detected by the RFID reader as a feedback and be shown on the PC screen and smart phone. The recorded images can be also transmitted back to the server via WiFi system for face tracking and discriminating analysis. On the other hand, the self-propelled vehicle patrolling routes can be modified by the Android smart-phone remote-control module. When some defined events occur, the build-in MSN module will notice users by sending messages to PC and smart phone. Experimental results are given in the paper to validate its performance.

\section{Introduction}

As the incidents of theft grew more frequent, the applications of security systems are more popular than ever to prevent the damages caused by theft whether at home or elsewhere. The traditional security system gives some protection to the situation but still has some dead zone that cannot be monitored. Therefore, this paper proposes mobile security monitoring system to improve the security of traditional one. The comparison diagram between the proposed and traditional security system is shown as Figures 1 and 2. A selfpropelled patrolling vehicle acts as a security patroller in the security system, which can monitor those dead zones of the traditional fixed surveillance system. The remote monitoring capabilities can also be enhanced by using the wireless network. And the face detection system is adapted to record and analyze the invaders [1-3]. No matter where the user is, he can monitor the indoor status by using network. There are also many literatures concerning about surveillance issue.
In [4], a surveillance security robot team had been developed combining with human recognition by using RFID. A LAN-based building surveillance robot was also proposed to secure the safety of a building [5]. Chen and Luo introduced the remote-control mobile robot via World Wide Web (WWW) [6]. And the proposed surveillance robot not only patrols the designed route automatically but also equips with the ability of face detection. By using the Internet, any invader will be noticed by the system via MSN or smart phone and furthermore control the patrol route of the surveillance vehicle.

OpenCV is an open source and cross-platform libraries, it can be used in most of the platforms such as the operating system of Linux and Windows [7]. OpenCV is developed by the Intel Corporation for image processing and providing interface to create pictures by $\mathrm{C}$ programming language and so on. It can be used to handle object tracking, face recognition, texture analysis, image file formats integrated with different matrix operations for the static image files, 


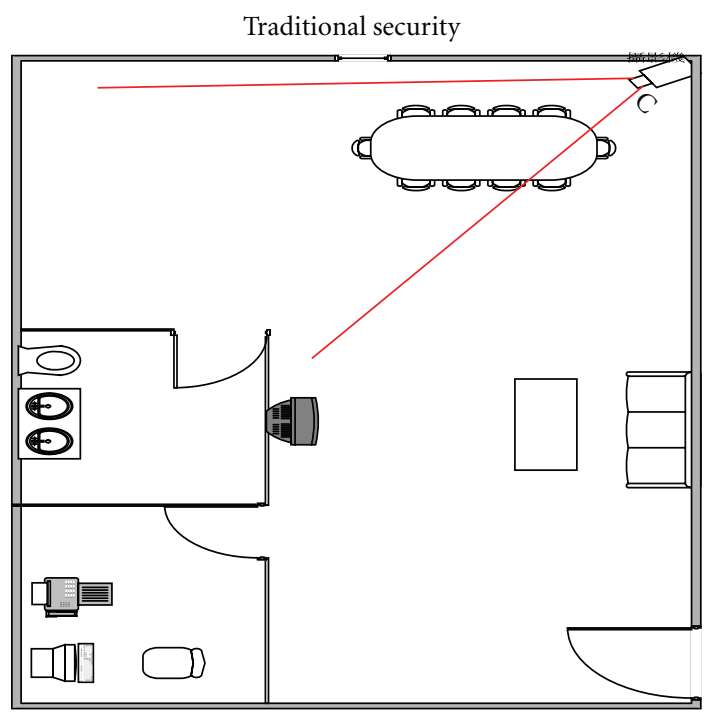

FIGURE 1: The traditional security system.

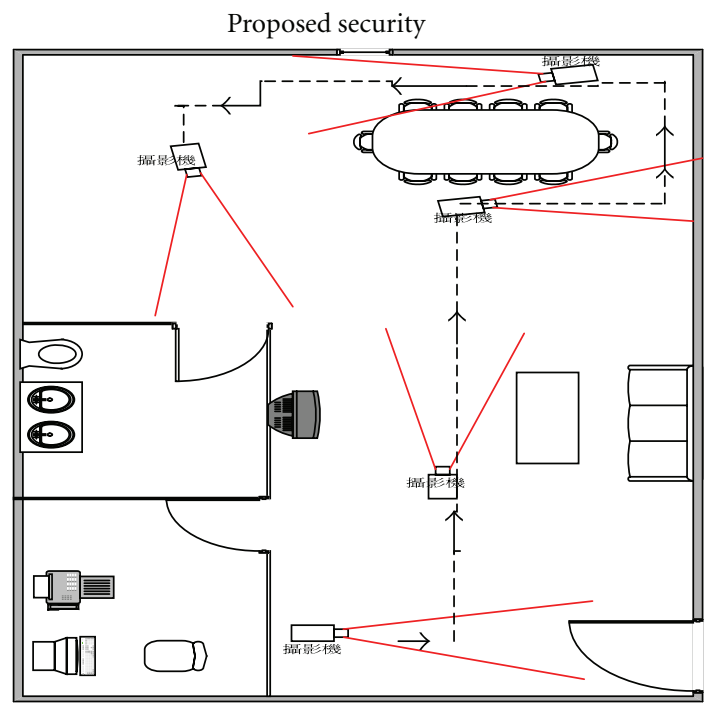

FIGURE 2: The proposed security system.

and the dynamic image processing of IPCAM [8]. In this paper, OpenCV technology is used for face detection. RFID system identifies the object by using the radio frequency technology to sense with small IC chip attached on the object $[9,10]$. In this way, RFID system can be used to track object by processing of a noncontact, short-range automatic identification technology. The RFID system is adopted in the proposed scheme, system components including tags, reader, and the host computer. Tag is a data storage device; the Reader reads information from the Tag. The reader reads the data and sends to the host computer for further processing. When sensing the radio waves emitted by the reader, the tag will produce a "magnetic induction" to trigger the RF transmitter module to send the built-in EEPROM information back to the reader. The reader is transmitted the information to host computer through the RS-232, or USB interface. There are two kinds of RFID tags such as active and passive. The passive type is chosen in the proposed scheme.
With the proposed combined scheme of the paper, the security of indoor surveillance will be upgraded. The selfpropelled vehicle will give more information than the traditional security system. Experimental results are also provided to validate the performance of the proposed system.

\section{System Architecture}

The proposed self-propelled monitoring and surveillance vehicle can be divided into the following parts: wireless IPCAM video capture system, face detection system, remote monitor and alarm transmitter system, RFID position detection systems, and cell phone monitoring and control system [11-13]. The diagram of system architecture is shown in Figure 3.

The self-propelled vehicle uses RFID technology to control the moving direction. RFID tag is installed in the righthand side of the self-propelled vehicle. When the self-propelled vehicle moves to a predefined routing path install-ed with RFID reader, the RFID reader would detect the RFID tag and send the signals back to the server to show the detected position on the map to indicate the status of the self-propelled vehicle. Smartphone (Android) can also send control command through server to control the direction of self-propelled vehicle. Face detection subsystem uses the Intel's OpenCV library to detect face of the monitored place $[14,15]$. There are two wireless IPCAMs mounted on the self-propelled vehicle to monitor the front and back of the vehicle for face detection. If a face was detected in the image file, the server would trigger the MSN robot to send warning message to the user $[16,17]$. Users can use the PC, notebook, or smart phone to monitor the situation or drive the selfpropelled vehicle to the spots where the users want it to be. The system description is shown as Table 1 .

The server provides the remote monitor website system which is installed with Microsoft IIS and the ASP.NET web program. Users can use the PC, notebook, and smartphone to connect this website for monitoring the place via wireless IPCAM. The RFID position detection system as shown in Figure 4, the RFID tag, is mounted on the right-hand side of the self-propelled vehicle. By the detecting of tag, a predefined routing path can be traced. The detected information is also displayed on the map of the monitored place. The server would also send guidance control command to the selfpropelled vehicle for the next position of the RFID reader.

The self-propelled vehicle is controlled through the WIFI module by receiving socket data from the server. The control center of the self-propelled vehicle is the DFRduino RoMeo 328 microcontroller [18]. The microcontroller receives control command through the socket data of the server to control the motion of self-propelled vehicle. Generally, the patrolling path of the self-propelled vehicle is predefined by installing the RFID tags in the proper positions as the predefined patrol route. If the warning message is detected, users can guide the self-propelled vehicle by remote control through smartphone as shown in Figure 5. If the RFID tags are not read in a time interval or the messages of RFID reader are not received by the server, then the system will notice 


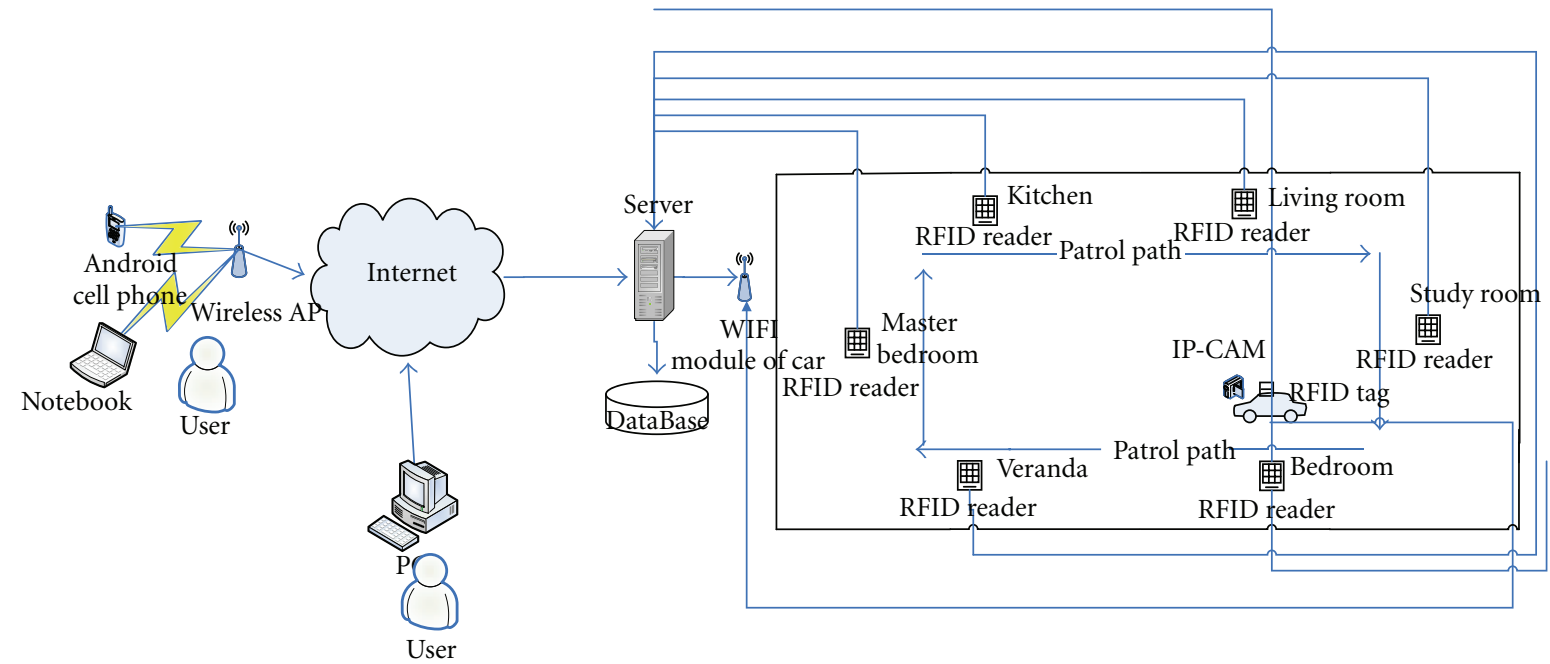

FIGURE 3: The architecture of the proposed system.

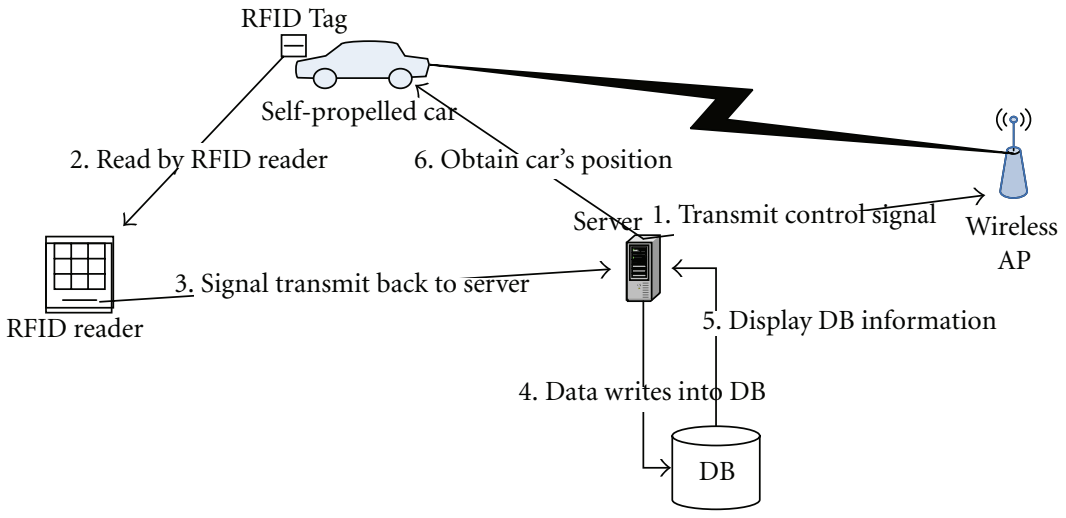

FIgURE 4: RFID position detection system.

the user to take actions to increase the reliability of the overall system.

\section{The Hardware Architecture of the Vehicle}

Figure 6 shows the hardware architecture of the self-propelled vehicle. As previously mentioned, the microcontroller of the self-propelled vehicle is DFRduino RoMeo 328. There are 14 sets of digital I/O interface(including 6 sets of PWM output), 8 sets of emulating analog I/O interfaces, 2 pairs of DC motor drives, and 6 input buttons in the Atmega168 based microcontroller.

The bottom layer of the self-propelled vehicle including four 3V DC motors, DFRduino RoMeo 328 microcontroller, WiFi module, RFID tag, and charger interface is shown as Figure 7. When the self-propelled is going to run out of power, it would detect the position of charger and go to it automatically for charging. The WiFi module receives the control command from the server or smartphone. The RFID tag in the self-propelled vehicle is used to detect its own position in the routing path and display the position of the self-propelled vehicle on the server.

The first layer of the self-propelled vehicle is the electricity detection PCB and power supply module as shown in Figure 8 . The power supply module used the $12 \mathrm{~V}$ battery to provide the power for two D-LINK wireless IPCAM $(5 \mathrm{~V}$ 1.2 A) and the DFRduino RoMeo 328 microcontroller(12 V). The electricity detection PCB is to detect the battery status to show the result by displaying the LED in red/green light.

The second layer of the self-propelled vehicle is the battery, PCB, and two D-LINK wireless IPCAM as shown in Figure 9. The regulator mounted on PCB should guarantee the steady output power of $12 \mathrm{~V}$. Therefore, the regulator on $\mathrm{PCB}$ can prevent the power from the charger to damage components in the self-propelled vehicle.

The third layer of the self-propelled vehicle is simply the solar panel. The realized implementation of self-propelled vehicle is shown as Figure 10. The vehicle equips with $4 \times 3 \mathrm{~V}$ DC motors which are controlled by the control module DFRduino Romeo 328 for driving the robot and 


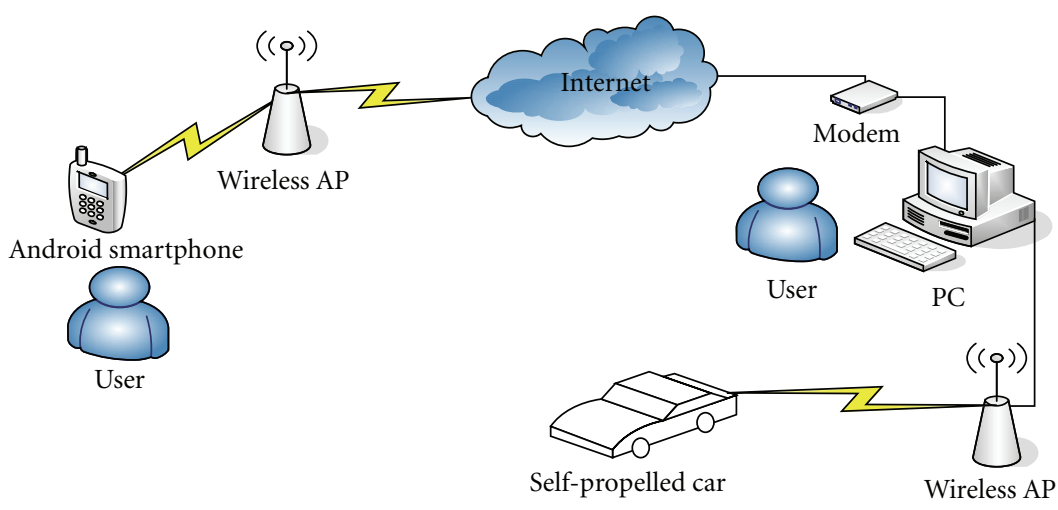

FIGURE 5: Smartphone control system.

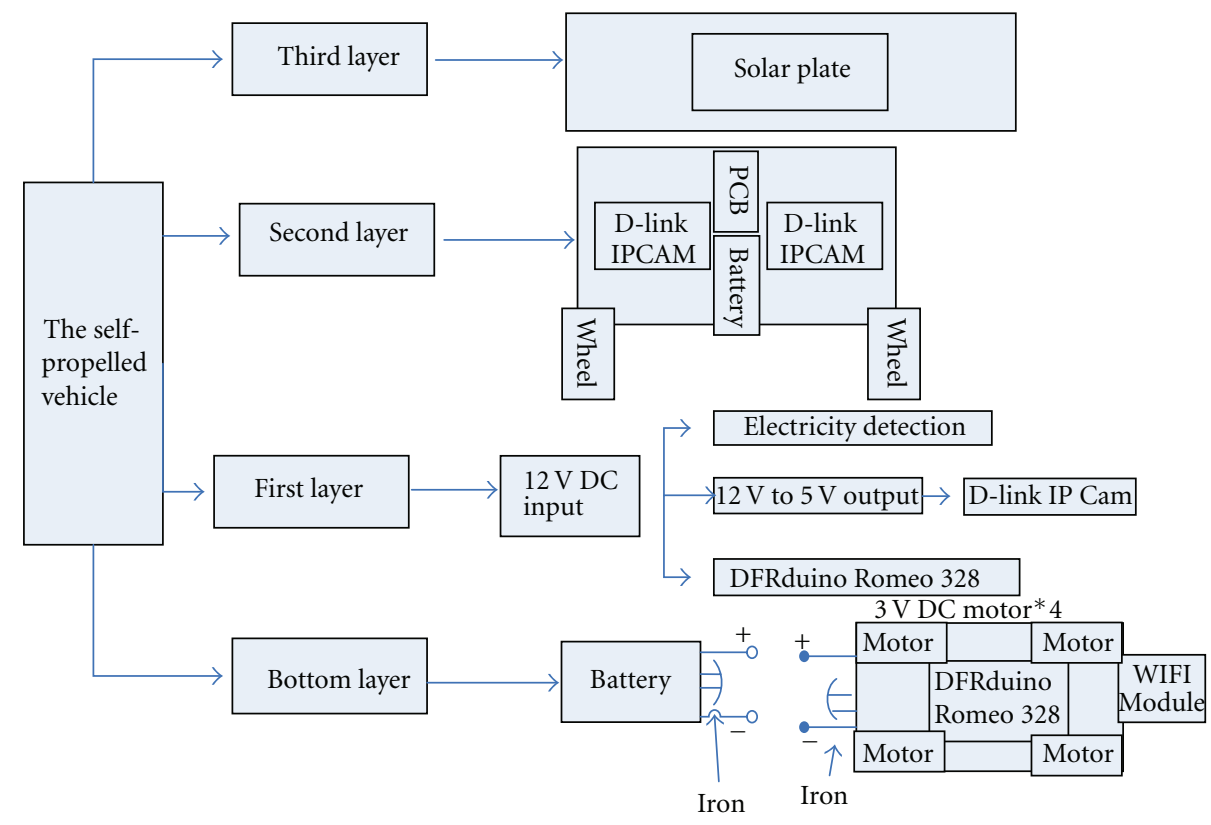

FIGURE 6: The hardware architecture of the self-propelled vehicle.

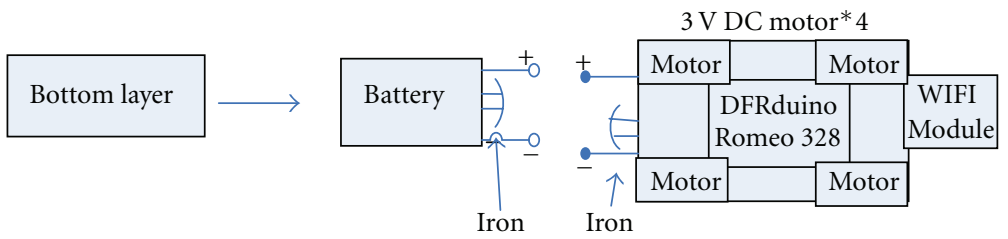

Figure 7: The sketch of bottom layer.

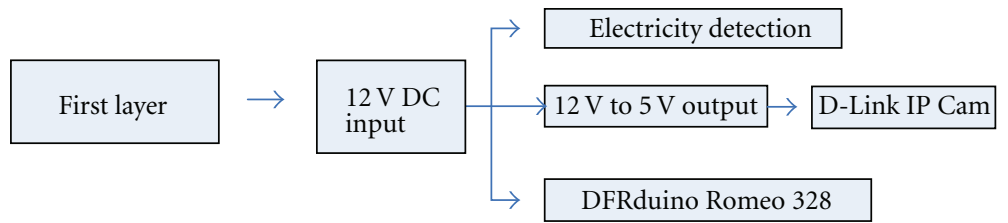

FIGURE 8: The sketch of first layer. 


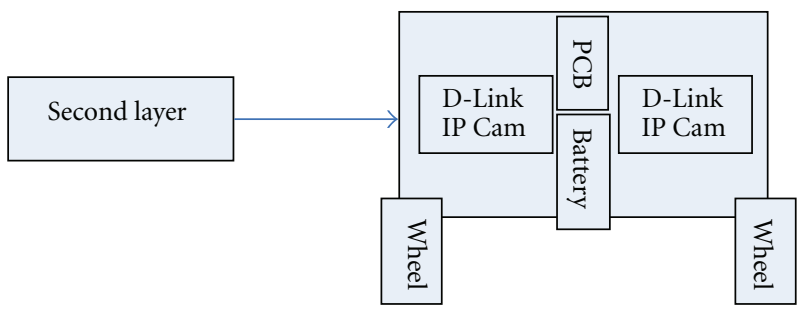

Figure 9: The sketch of second layer.

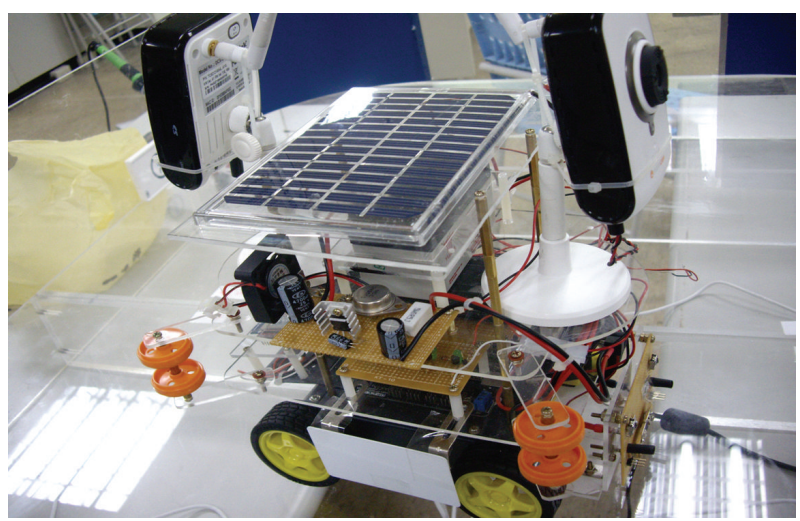

FIgURE 10: The implementation of the self-propelled vehicle.

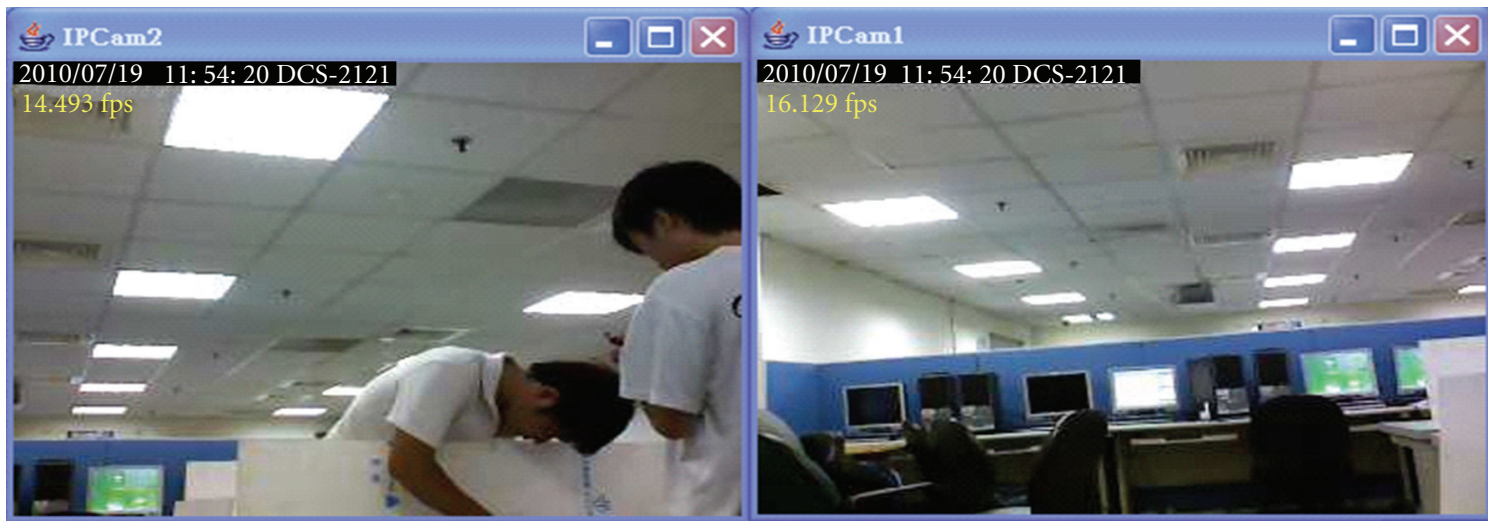

FIGURE 11: Display result of local wireless IPCAM (left: front; right: rear).

TABLE 1: Subsystem functional description.

\begin{tabular}{ll}
\hline Subsystem & Function description \\
\hline Wireless IPCAM video capture system & $\begin{array}{l}\text { Accordance to the temporary path provided by the manufacturer to capture the pictures } \\
\text { through wireless IPCAM and convert into image files }\end{array}$ \\
\hline Face detection system & Face detection for each captured image files \\
\hline Remote monitor and alarm transmitter system & Transmit warning message to user through MSN robot \\
\hline RFID position detection systems & $\begin{array}{l}\text { Remote monitoring function of watching the indoor status, self-propelled vehicle, and } \\
\text { the newest detected face }\end{array}$ \\
\hline Self-propelled vehicle & $\begin{array}{l}\text { Reading the RFID tag of self-propelled vehicle and displaying the car position by the } \\
\text { installed RFID reader; these information is recorded in the server database }\end{array}$ \\
\hline Smartphone monitoring and control system & $\begin{array}{l}\text { The server controls self-propelled vehicles through the wireless network } \\
\text { the Android mobile phone }\end{array}$ \\
\hline
\end{tabular}




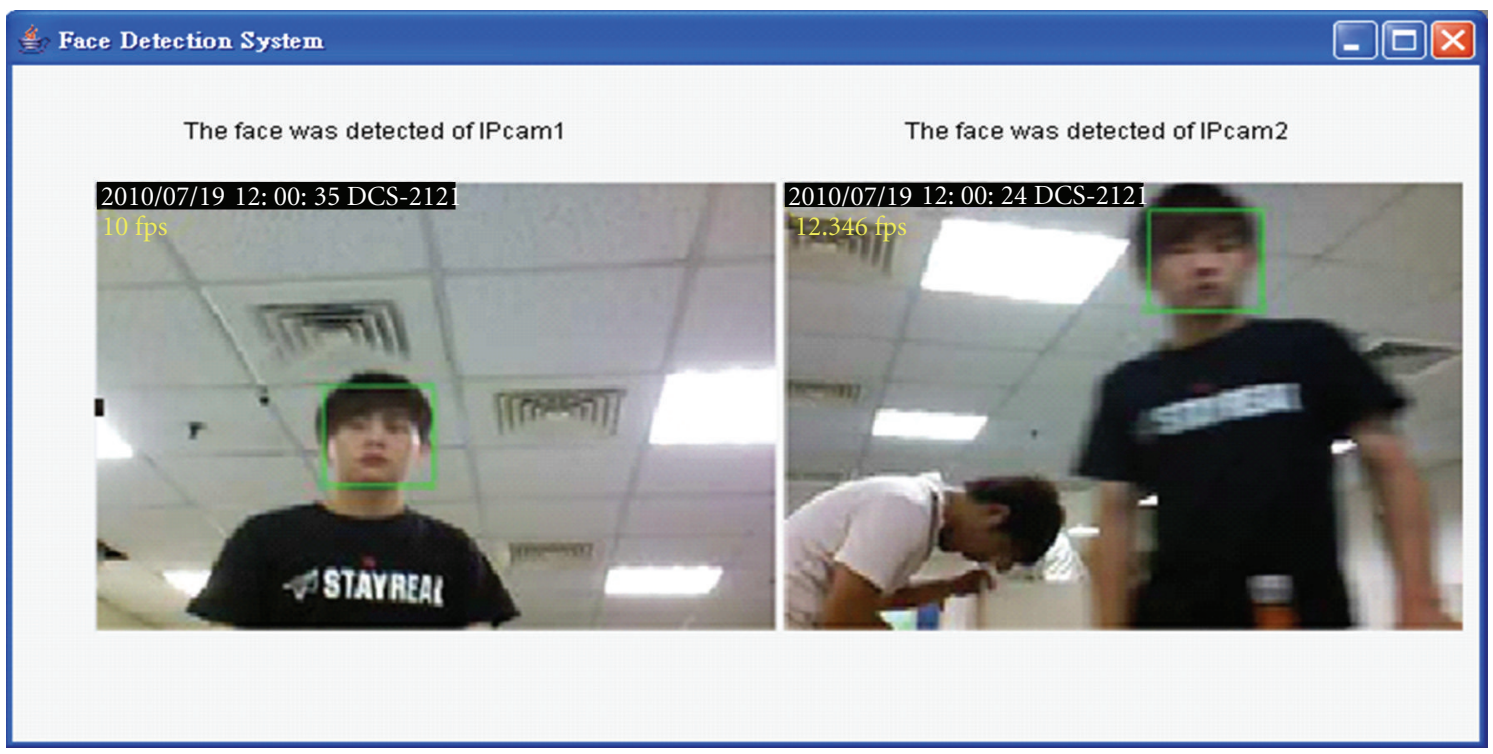

FiguRE 12: Face detection application program.
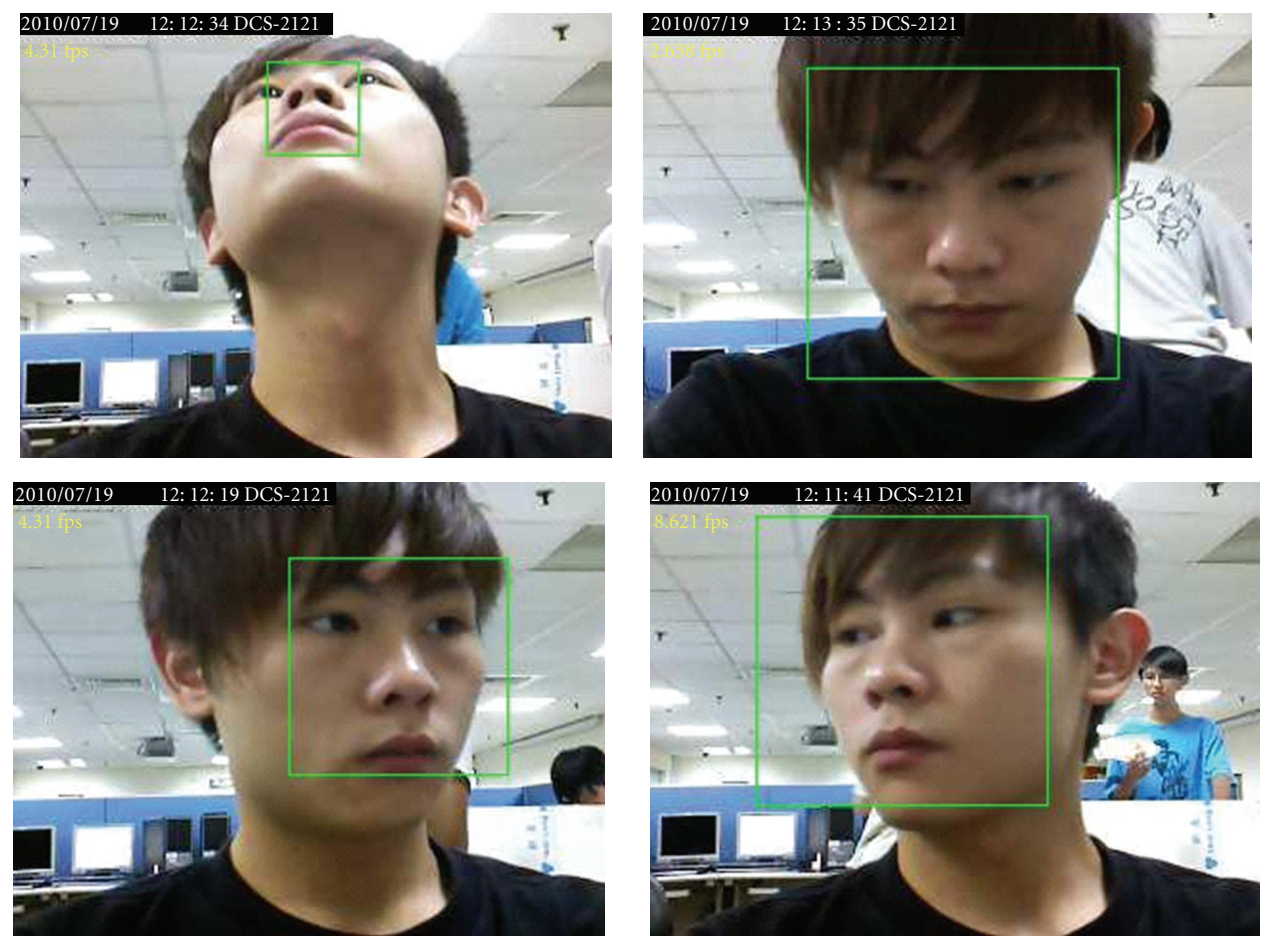

FIGURE 13: The limit angle of face detection.

a power supply of a $12 \mathrm{~V}$ battery. Its approximate size is $200 \times 160 \times 450 \mathrm{~mm}$ with IPCAM.

\section{Experimental Results}

The wireless IPCAM mounted on the self-propelled vehicle should be specified IP by log in the account and password provided by the manufacturer. When the self-propelled vehicle is patrolling, the picture would be captured and stored in the image files in the temporary memory of the IPCAM, respectively. The server then gets the image files via WiFi system and shows them on the display as shown in Figure 11.

Figure 12 shows the face detection application program implements by JNI provided by OpenCV [14]. There are 100 pictures used to test the face detection. Bigger size of the search window would increase the failure rate of face detection. The resolution of the picture is also another reason 


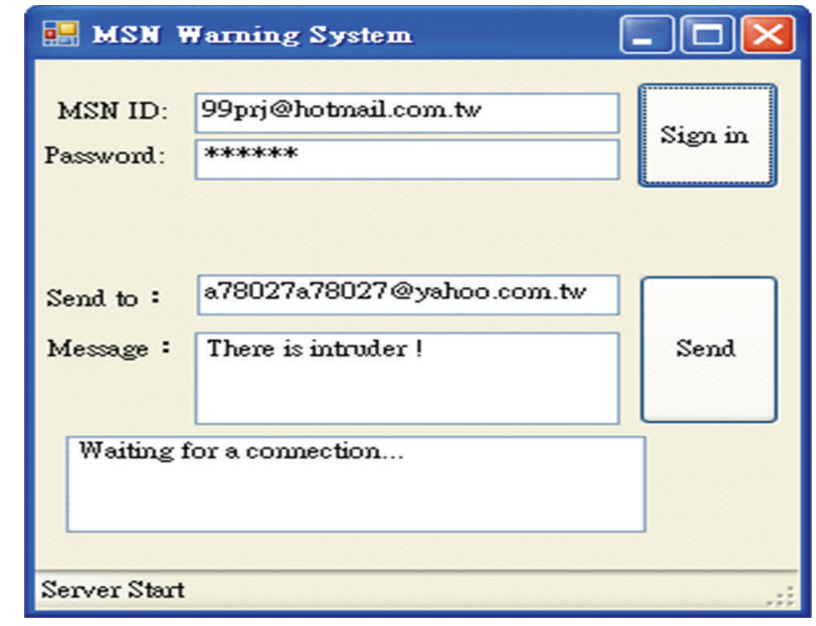

FIGURE 14: The warning message of face detection via MSN.

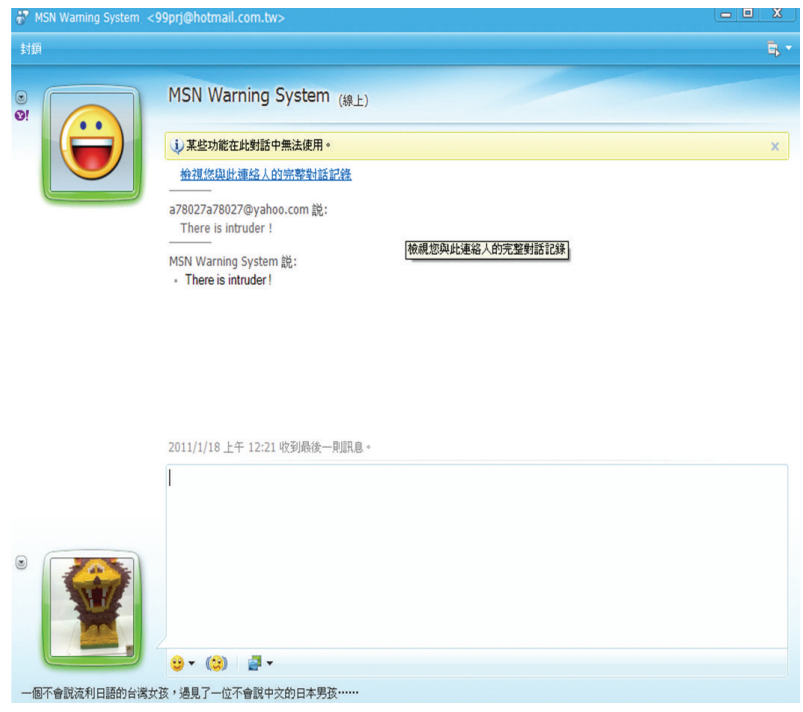

(a) Warning message on MSN

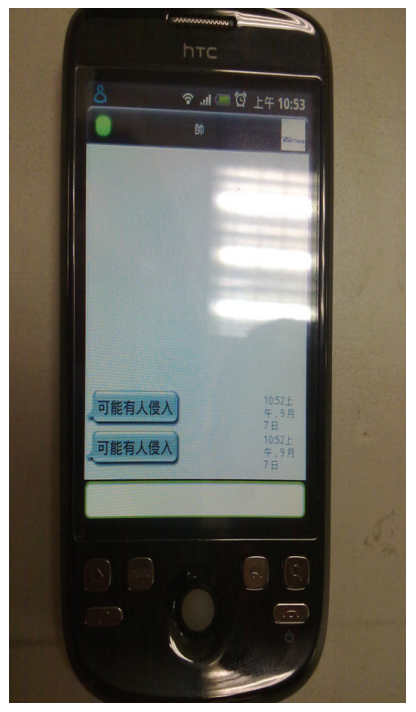

(b) Warning message on smart phone

Figure 15: Automatic status report.

TABle 2: The distance and image limit of face detection.

\begin{tabular}{lccccc}
\hline Image size & $\begin{array}{c}\text { Distance } \\
\text { between } \\
\text { face and } \\
\text { IPCAM }\end{array}$ & $\begin{array}{c}\text { Limit of } \\
\text { face up } \\
\text { angle }\end{array}$ & $\begin{array}{c}\text { Limit of } \\
\text { face } \\
\text { down } \\
\text { angle }\end{array}$ & $\begin{array}{c}\text { Limit of } \\
\text { face turn } \\
\text { left angle }\end{array}$ & $\begin{array}{c}\text { Limit of } \\
\text { face turn } \\
\text { right angle }\end{array}$ \\
\hline $640 * 480$ & $<4.20 \mathrm{~m}$ & $30^{\circ}$ & $15^{\circ}$ & $30^{\circ}$ & $30^{\circ}$ \\
$320 * 240$ & $<2.25 \mathrm{~m}$ & $30^{\circ}$ & $15^{\circ}$ & $30^{\circ}$ & $30^{\circ}$ \\
\hline
\end{tabular}

for face detection. The higher resolution would increase successful rate of face detection. The test environment is indoor, light source is fluorescent light, and input file of image resolution is $320 * 200$. The test result shows that when the left/right angle of face and IPCAM is greater than 30 degrees, the face detection method cannot detect any face.
When the down angle of face and IPCAM is greater than 15 degrees and the up angle of face and IPCAM is greater than 30 degrees, then the face detection method cannot normally works. As the distance between image and IPCAM would also influence the resolution of face detection, 2.25 meter is the maximal distance. If the resolution is increased to $640 * 480$, the maximal distance between image and IPCAM is 4.2 meter. Figure 15 is the test result of the limitations shown in Table 2 with the testing process shown in Figure 13.

When a face is detected, the MSN system will notify the user by sending messages by MSN as shown in Figure 14. Because the vehicle is moving with front and rear IPCAM, the restriction of face detection may expand to a wider range in reality. A modified program is given to notify the user if any moving object is detected that will increase the reliability of the system. 
The position of self-propelled vehicle

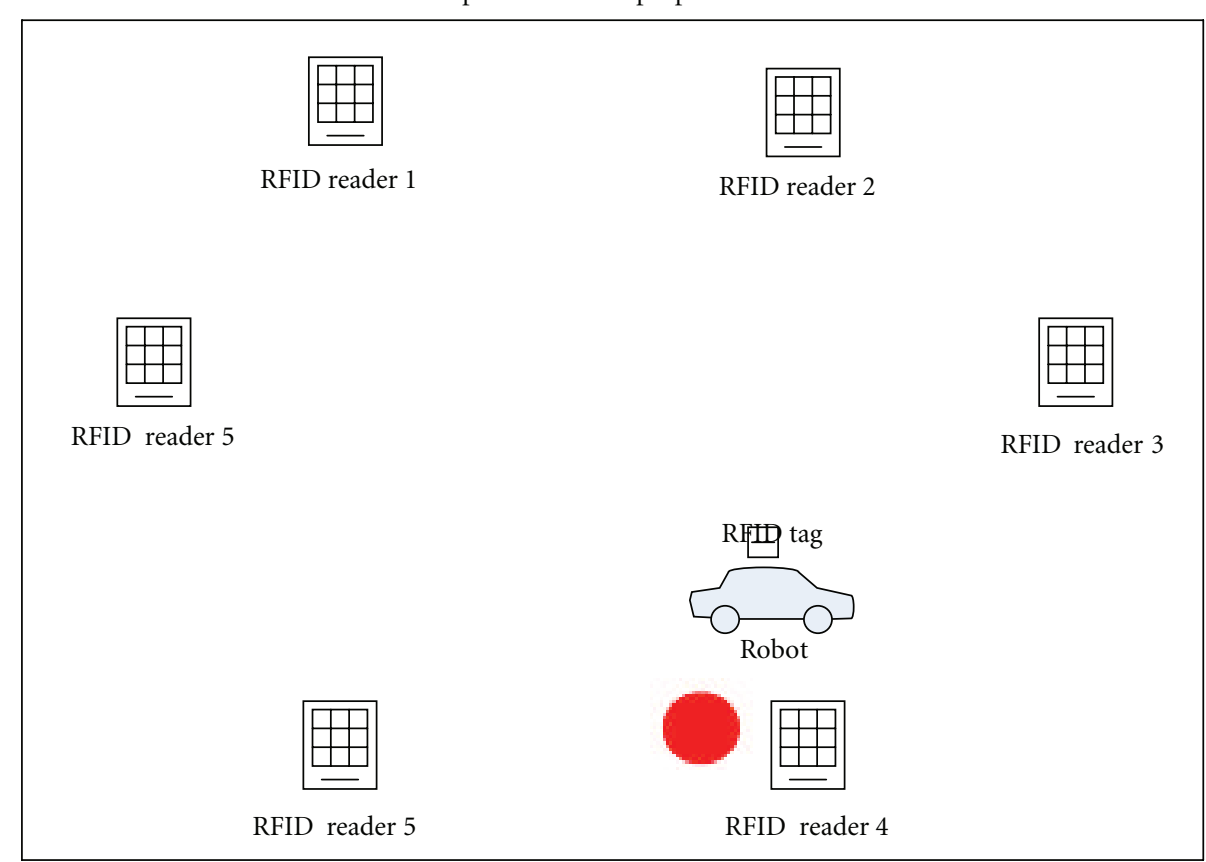

(a) The display on monitor

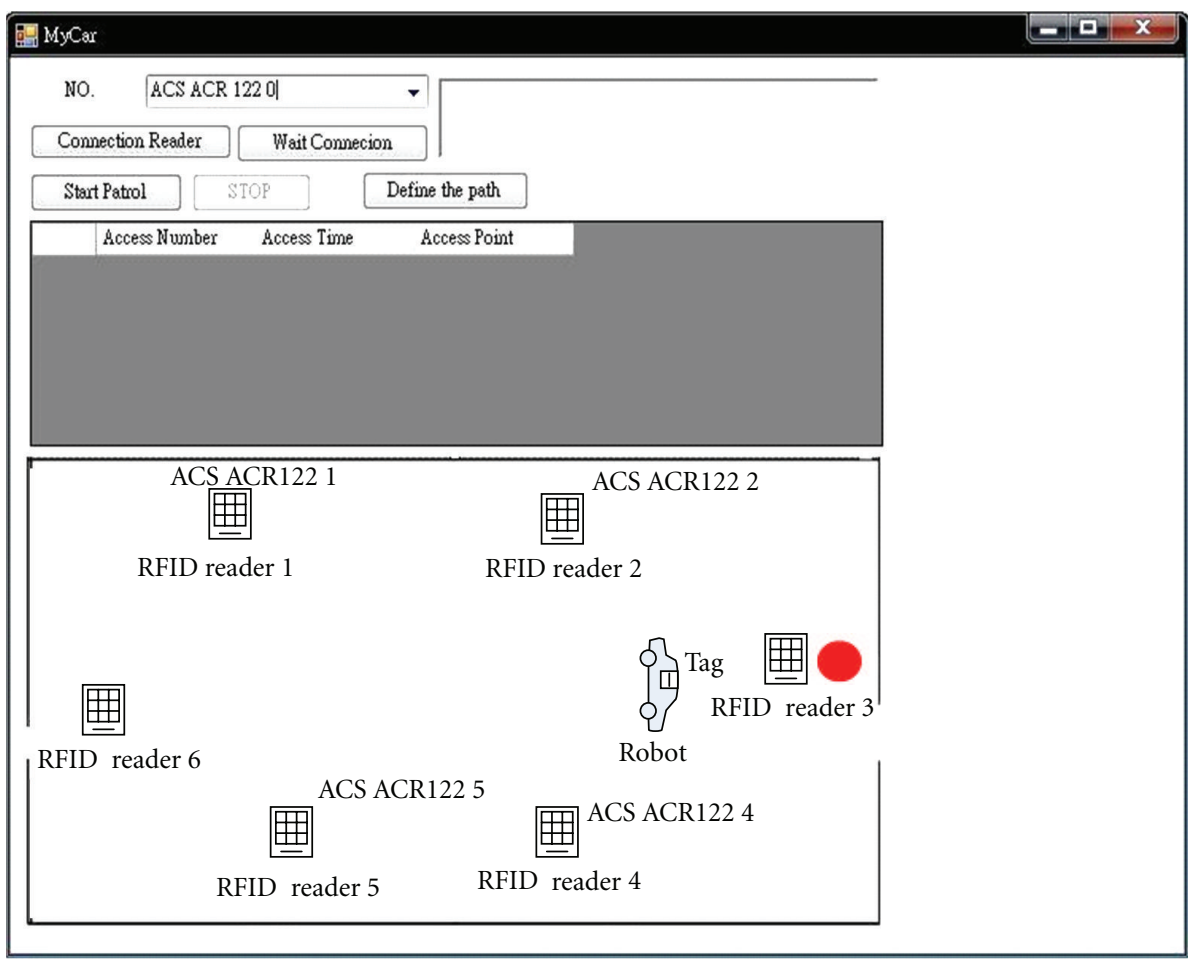

(b) The display on smart phone

FIGURE 16: The position of the self-propelled vehicle.

The tag of self-propelled patrol vehicle would be detected by different RFID readers mounted on the patrolling path to guide the vehicle. As the self-propelled vehicle being detected by the RFID reader, this information would be sent to server that records the time and position simultaneously. And the position of the vehicle will be marked on the map and displayed on the monitor or smartphone as shown in Figures 16(a) and 16(b), respectively. 


\section{Conclusion}

The proposed system is implemented on a PC server, self-propelled vehicle, and some small smart systems via networks to provide the functions of surveillance and remote control. The proposed surveillance robot using RFID technique to guide the vehicle cruising according to the predefined route. A face detection technique is adopted in this paper by using IPCAM to find out the invader. The WIFI is also applied here to not only transmit the messages from RFID reader and IPCAM or the warning messages to user but also send the remote control signals to the vehicle if necessary. To summarize the proposed system, there are some points that can be addressed as follows:

(i) the wireless IPCAM being equipped within a mobile vehicle to obtain wider range of monitoring;

(ii) the warning message can be back to the security center and/or the user by MSN and/or smart phone;

(iii) face detection technique is adopted in the proposed system to identify the invader;

(iv) the self-propelled vehicle can be navigated by MSN and/or smart phone if necessary and displayed its position by RFID readers mounted on the patrolling route;

(v) the self-propelled vehicle can automatically charge itself as the battery low being detected.

The proposed indoor security system is developed with some proper design described in the previous section. With experimental results, the feasibility of the proposed scheme is validated.

\section{Acknowledgment}

This paper is partially sponsored by the project of NSC 992221-E-147-004- and NSC 100-2221-E-147-001-in Taiwan.

\section{References}

[1] C.-Y. Wang, Utilization Of Deformable Templates In Real-Time Face Tracking System, M.S. thesis, National Sun Yat-sen University, Taiwan, China, July 2007.

[2] Y.-Z. Xie, A Study of Real-Time Face Tracking with an Active Camera, M.S. thesis, National Sun Yat-sen University, Taiwan, China, April 2005.

[3] V. Paul and J. J. Michael, "Rapid object detection using a boosted cascade of simple features," in Proceedings of the IEEE Computer Society Conference on Computer Vision and Pattern Recognition (CVRP '01), vol. 1, pp. 511-518, Hawaii, USA, December 2001.

[4] T.-H. S. Li, C.-Y. Chen, Y.-C. Yeh et al., "An autonomous surveillance and security robot team," in Proceedings of the IEEE Workshop on Advanced Robotics and its Social Impacts (ARSO '07), pp. 1-6, Hsinchu, China, December 2007.

[5] A. T. P. So and W. L. Chan, "LAN-based building maintenance and surveillance robot," Automation in Construction, vol. 11, no. 6, pp. 619-627, 2002.

[6] T. M. Chen and R. C. Luo, "Remote supervisory control of an autonomous mobile robot via world wide web," in Proceedings of the IEEE International Symposium on Industrial Electronics Conference (ISIE '97), vol. 1, pp. 60-64, 1997.

[7] OpenCV, http://www.opencv.org.cn/index.php.

[8] MJPG raw streaming video from IPCAM, http://forums.sun .com/thread.jspa?threadID $=494920$ \&messageID $=4078640$.

[9] Introduction to the RFID, http://www.cc.ntu.edu.tw/chinese/ epaper/0002/20070920_2005.htm.

[10] Comparie RFID with other system, http://www.yeon.com.tw/ content/techinfo.php?c_id=16.

[11] Basic Concept of Android System, http://developer.android .com/index.html.

[12] Using the Eclipse Environment to develop Android Application program, http://blog.yslifes.com/archives/279/commentpage- 1 .

[13] Android Socket[1], http://bbs.hiapk.com/thread-6210-1-1 .html.

[14] The use method of JNI, http://en.wikipedia.org/wiki/Java Native_Interface.

[15] G. Bradski, A. Kaehler, and V. Pisarevsky, "Learning-based computer vision with intel's open source computer vision library," Intel Technology Journal, vol. 9, no. 2, pp. 119-131, 2005.

[16] MSN messenger open-source class library, http://www .xihsolutions.net/dotmsn/index.html.

[17] MSNP-Sharp, http://huan-lin.blogspot.com/2008/11/msn .html.

[18] Arduino, http://www.arduino.cc/. 

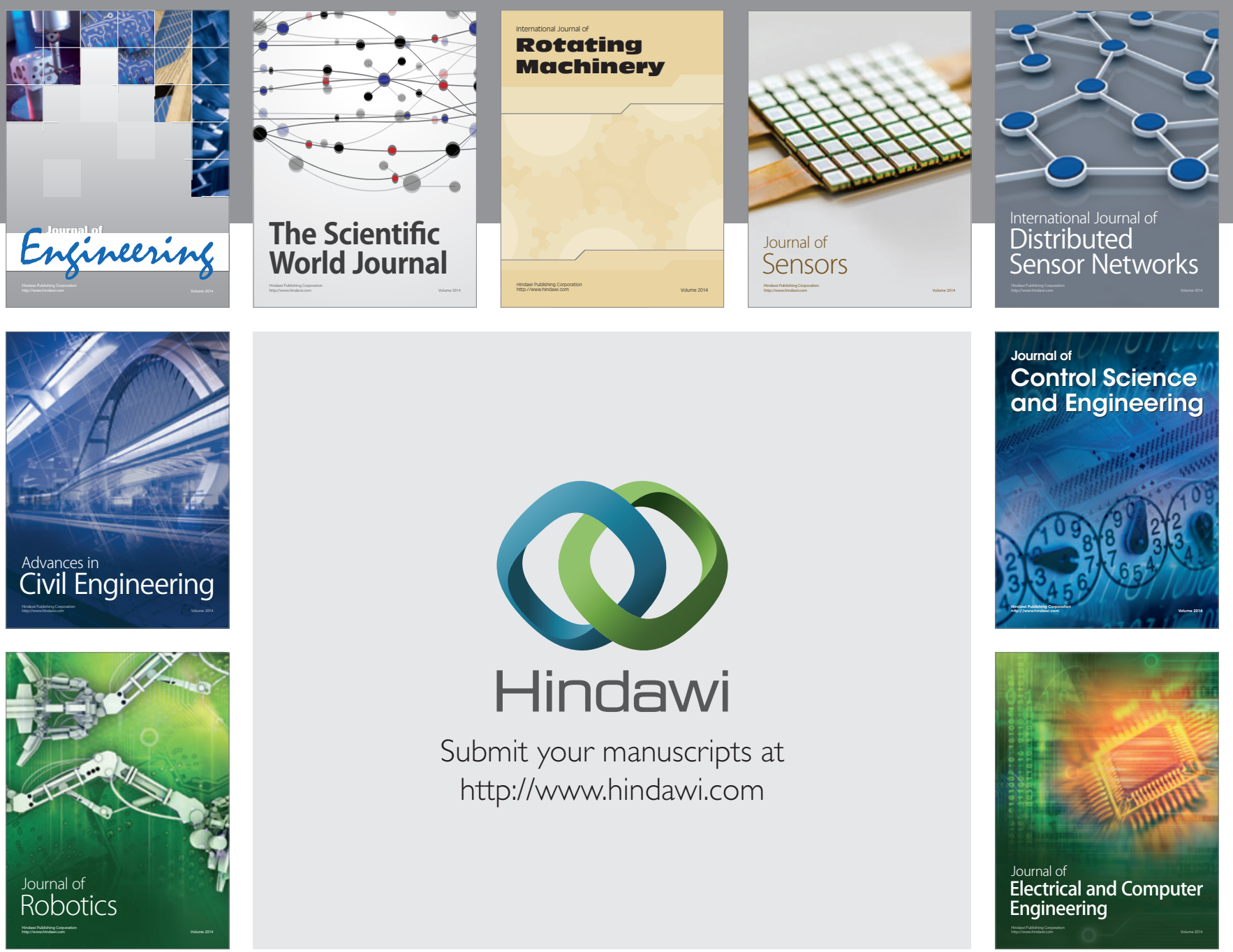

Submit your manuscripts at

http://www.hindawi.com
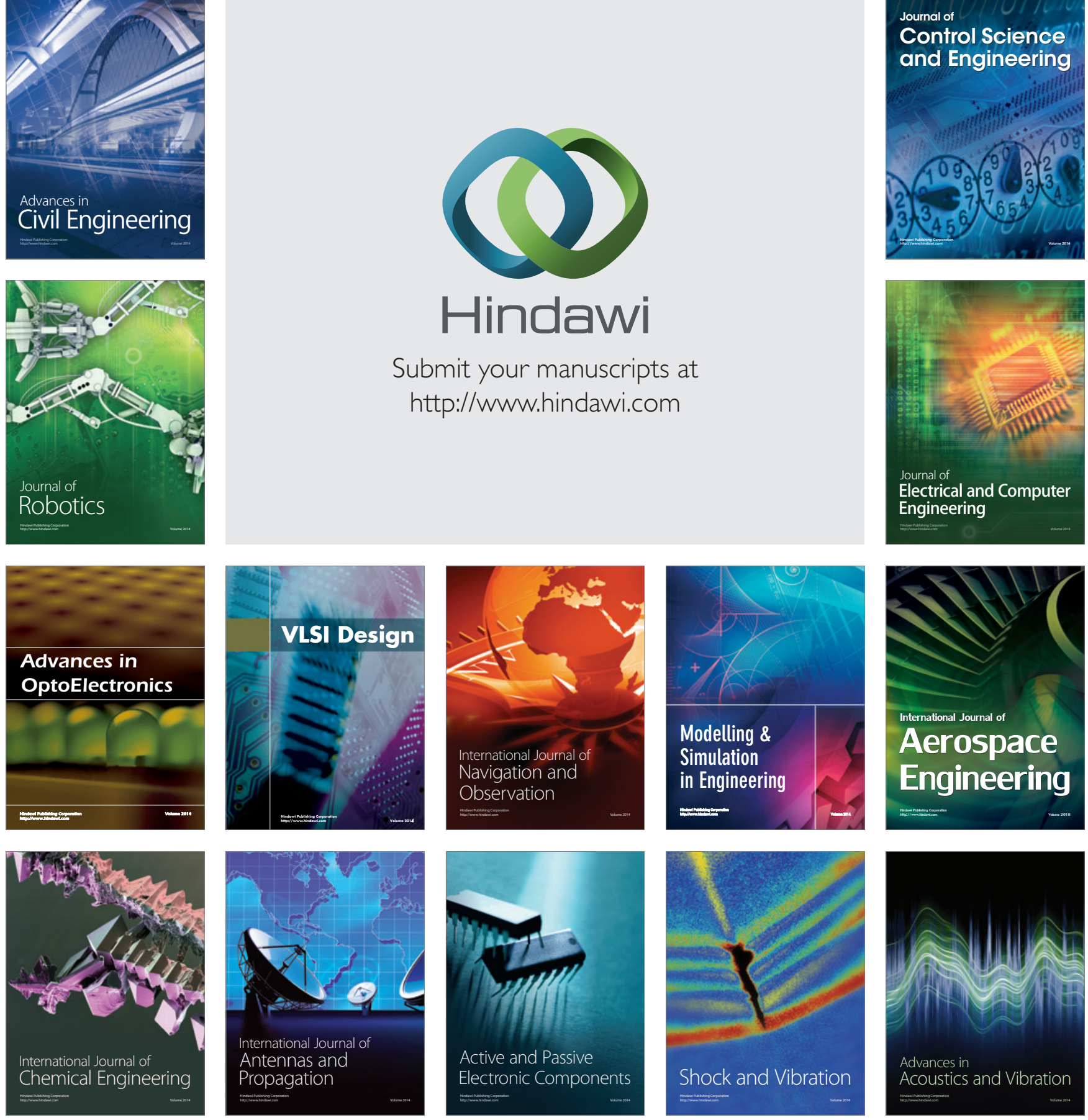